SCIO. Revista de Filosofía, n. ${ }^{\circ}$ 18, Julio de 2020, 149-175, ISSN: 1887-9853

\title{
IDENTIDAD PERSONAL Y SUJETO ÉTICO DOS MODELOS ALTERNATIVOS A DEREK PARFIT
}

\author{
PERSONAL IDENTITY, SUBJECT AND ETHICS \\ TWO ALTERNATIVE VIEWS TO DEREK PARFIT
}

Manuel Bares Partal ${ }^{*}$

Fechas de recepción y aceptación: 5 de junio de 2019 y 29 de abril de 2020

Resumen: La identidad personal es el tema central del pensamiento de Derek Parfit y su principal argumento en defensa de una concepción utilitarista de la ética. Desde su perspectiva, la identidad se resume en lo que llama Relación R de conectividad y continuidad psicológica. A través de su análisis de diversos experimentos mentales el autor llega a la conclusión de que la identidad no está determinada y, por lo tanto, no es "lo que importa". Lo que importa es la relación R. Sobre dicha relación y no sobre identidades concretas han de girar las decisiones morales. Sin embargo, existen diversos planteamientos críticos que lejos de suponer, en palabras de Parfit, un hecho suplementario, construyen un concepto de identidad no utilitarista. En este trabajo vamos a examinar la identidad narrativa en la obra de Paul Ricoeur, así como el planteamiento neokantiano de Christine Korsgaard.

Palabras clave: identidad personal, relación R, utilitarismo, identidad narrativa, agencialidad.

\footnotetext{
a Departamento de Humanidades Contemporáneas. Universidad de Alicante. IES Playa de San Juan de Alicante.

* Correspondencia: Universidad de Alicante. Edificio III de la Facultad de Filosofía y Letras. Carretera de San Vicente del Raspeig s/n. 03690 (San Vicente del Raspeig), Alicante. España.

E-mail: manuelbares@iesplayasanjuan.com
} 
Abstract: Personal identity is the main topic in Derek Parfi's works, and his principal argument for an utilitarian conception of ethics. According to his view, we can summarize identity in what he calls $\mathrm{R}$ relation, that is, psychological connectivity and continuity. Through his analysis of several mental experiments Parfit arrives to the conclusion that identity is not determined, so it is not what matters. What matters is the $\mathrm{R}$ relation, and it is about such a relation, and not about concrete identities, that moral decisions are concerned. Nevertheless, there are different conceptions of identity which are critical with Parfit's view and which are very far from what Parfit calls "the simple view". Most of them are critical with utilitarianism. In this essay I'm going to consider Paul Ricoeur's narrative identity, and Christine Korsgaard's neokantian view.

Keywords: personal identity, $\mathrm{R}$ relation, utilitarianism, narrative identity, agency.

\section{$\S 1$ INTRODUCCIÓN}

Imaginemos que una persona joven, un niño con cierto carácter filosófico, comienza a preguntarse por su futuro. Tanta molesta insistencia de los adultos en preguntar qué quiere ser de mayor le hace plantearse, quizás como juego, realmente quién llegará a ser en el futuro. Trata de imaginar cómo será su proyecto de vida futura. ¿Qué conexiones y recuerdos mantendrá después de tanto tiempo? ¿Acaso olvidaría tantas cosas como sabe ahora y se volvería tan aburridamente previsible como todos los adultos? No le gusta la idea. Quiere evitarlo a toda costa y decide escribir una carta, con "todo lo que importa", a su yo futuro con una advertencia en el sobre. "Para mí mismo. No abrir hasta ser mayor".

Imaginemos que ese niño ya es mayor y encuentra la carta. No recuerda lo que escribió ni en qué momento lo hizo, pero sabe que aquel niño ocurrente que le dejó un importante y simpático mensaje hace años es él mismo en el pasado y, por lo tanto, sabe que es a sí mismo a quien va dirigida. Por supuesto está la cuestión de cómo sabe que es la misma persona, pero lo cierto es que lo sabe. Averiguar qué mecanismos psicológicos y neuronales lo posibilitan es una cuestión que deben explicar los neurocientíficos. Pero la pregunta que 
se hace es aún más compleja: ¿qué quiere decir que es la misma persona?, ¿qué es lo que hace que ese niño, tan diferente al adulto que es ahora, sea él mismo? y, además, ¿cuáles son las condiciones que permiten que, a pesar de todos los cambios posibles a lo largo de una vida, esa persona permanezca? Estas dos cuestiones, que plantean las condiciones necesarias y suficientes de la identidad personal, tanto diacrónica como sincrónica, conforman uno de los problemas filosóficos más interesantes desde la modernidad; el problema del sujeto.

En este trabajo pretendo esbozar crítica y brevemente algunas de las aportaciones más importantes a este problema dentro de la llamada "tradición analítica" en filosofía. En concreto a aquellos autores que toman a Locke como principal antecedente. De todos ellos quizás el planteamiento más llamativo sea el de Derek Parfit. Son bien conocidos sus recursos a la ciencia ficción, así como sus tesis acerca de un yo no determinado y "lo que importa". Parfit justifica así un determinado modelo ético; el utilitarismo. Voy a tratar de mostrar el camino recorrido por Parfit en su reflexión para, posteriormente, confrontarla a dos planteamientos tan distintos como son el enfoque hermenéutico de la identidad narrativa de Paul Ricoeur y el planteamiento neokantiano de Christine Korsgaard. Por último, trataré de sacar algunas conclusiones críticas al respecto.

\section{§2. Planteamiento del problema}

Harold Noonan (1989) afirma en su libro dedicado a esta cuestión que, si bien buena parte de los problemas filosóficos a lo largo de la historia del pensamiento occidental no son más que notas a pie de página de los Diálogos de Platón, gran parte de la cuestión de la identidad personal son solo notas al pie del Essay de Locke. El concepto de sujeto como substancia pensante cartesiana ha de ser, para Locke, diferente del concepto de persona. No solo por su escepticismo acerca del sustancialismo cartesiano, sino por cuestiones prácticas que el propio Locke denomina "forenses". La concepción cartesiana de una substancia pensante separada e independiente del cuerpo que habita es para Locke insuficiente para explicar la relación de identidad de una persona. 
Locke relaciona directamente la relación de identidad con la conciencia que toda persona tiene de sus propios recuerdos respecto a los recuerdos de los demás. Es decir, hace depender la unidad de la persona en la unidad de la conciencia, y esta se le hace explícita al sujeto a través de la unidad de los estados de memoria de sus propias experiencias. Es dicha unidad la que le permite reconocerlas como suyas y a él mismo como la persona que las tiene. Admitir que somos una substancia pensante no garantiza que aquellos contenidos de mi conciencia que refieren a mis propias experiencias sean diferentes de mi acceso a otros tipos de estados mentales. La misma substancia pensante podría haber estado vinculada a otro ser humano, y conformar una persona completamente distinta.

Let anyone reflect upon himself and conclude that he has in himself an immaterial spirit...; let him also suppose it to be the same soul that was in Nestor at the siege of Troy; but he now having no consciousness of any of the actions... of Nestor...does he or can he conceive himself the same person with him? Can he be concerned in his actions, attribute them to himself, or think them his own, more than the actions of any other man that ever existed? No more than if some of the particles of matter that were once a part of Nestor were now a part of this man: the same immaterial substance, without the same consciousness, no more making the same person...than the same particle of matter, without consciousness, makes the same person ${ }^{1}$.

La solución que aporta en este texto señala a un concepto de persona identificable y concreto, y no una substancia espiritual definida en términos universales como "res cogitans". Así, Locke entiende que la persona es ese ser:

Which is sensible or conscious of pleasure and pain, capable of happiness or misery, and so is concerned for itself as far as that consciousness extends ${ }^{2}$.

${ }^{1}$ Locke, Essay II, xxvii, 14.

${ }^{2}$ Locke, Essay II, xxvii, 17. 
No puede responsabilizarse a una substancia pensante de acción alguna, pero sí a una persona como la que Locke presenta. Lo que vincula a un sujeto con sus estados de conciencia pasados es la memoria experiencial, y en ella hace descansar Locke el peso de la identidad. Al mismo tiempo, para Locke, la unidad con los estados de conciencia anteriores fundamenta el derecho entendido como un sistema de justicia basado en la eficacia del castigo y la recompensa.

In this personal Identity is founded all the right of reward and punishment; happiness and misery being that for which everyone is concerned for himself... ${ }^{3}$

Esta manera de entender la identidad es, como hemos recordado al principio de este apartado, la piedra de toque de toda concepción que sobre este tema ha venido defendiéndose en la tradición anglosajona. Se puede aceptar el planteamiento lockeano o ser crítico con él, pero no puede pasarse por alto su contribución en esta cuestión. Sin embargo, la filosofía actual debe antes enfrentar algunas cuestiones previas.

La estrecha relación entre identidad y memoria hace que Locke tenga que defender a veces intuiciones que hoy nos parecen extrañas. Si alguien comete un delito y demuestra no recordar haberlo cometido no podríamos juzgarlo pues, si bien puede tratarse del mismo "hombre", no podemos demostrar que se trate de la misma persona. Una curiosa ontología en la que aparecen disociadas la sustancia pensante, la persona y el ser humano. Dicha ontología es difícil de comprender desde los principios de la actual filosofía de la mente. Existe el olvido, y parece lógico pensar que quien olvida es la misma persona que recordaba.

Además de esta perplejidad, las críticas a la unidad de la memoria en Locke no tardaron en aparecer y, curiosamente, son las mismas críticas que preocupan y determinan aun hoy a los defensores del llamado criterio psicológico. Así, Joseph Butler opondrá a Locke su famoso argumento de circularidad ${ }^{4}$. Según dicho argumento, cuando Locke hace depender la unidad de la conciencia de

\footnotetext{
${ }^{3}$ Locke, Essay II, xxvii, 18.

${ }^{4} \mathrm{El}$ argumento original aparece en "Of personal Identity". En The Analogy of Religion, 1736.
} 
la capacidad de memoria experiencial del sujeto, este debe ya hacerse consciente de sus experiencias pasadas "como suyas". Es decir, la memoria no es un hecho primitivo sino derivado del sujeto que las tiene. Ahora bien, si para acceder a la memoria pasada hay que hacerlo como sujeto, como unidad en la que este o aquel recuerdo han de acomodarse, entonces dichos recuerdos no pueden ser determinantes de la persona, pues la presuponen.

Otra objeción clásica e importante es la conocida crítica de vulneración de la transitividad que hace T. Reid ${ }^{5}$. Según este argumento es lógico pensar que si una persona $\mathrm{P} 1$ en t1 es idéntica a una persona $\mathrm{P} 2$ en $\mathrm{t} 2$, y esta a su vez es idéntica a otra persona $\mathrm{P} 3$ en $\mathrm{t} 3$, entonces hemos de concluir que P1 es idéntica a P3, pues de lo contrario romperíamos la ley de transitividad lógica. Ahora bien, según el criterio de Locke es posible que P3 tenga recuerdos de $\mathrm{P} 2$ pero no de $\mathrm{P} 1$, al tiempo que $\mathrm{P} 2$ sí conserva aún recuerdos de $\mathrm{P} 1$ en $\mathrm{t} 1$. Por lo tanto deberíamos colegir que P1 es la misma persona que P2 pero distinta de P3, al tiempo que P2 y P3 sí son la misma persona, lo que es inconsistente.

Cuestiones acerca del olvido, de la circularidad y de la transitividad han sido tratadas por distintos autores contemporáneos de diversas maneras. No sería muy aventurado decir que algunas posiciones respecto al problema que nos atañe se construyen alrededor de su respuesta a alguna o varias de estas críticas.

\section{§3. El CRITERIO PSicolóGico de LA IDENTIDAd PERSONAL. ReSPuestas A LAS CRÍTICAS CLÁSICAS}

Como decíamos, buena parte de los autores del mundo anglosajón se adhieren a la explicación lockeana de la identidad personal. Según la mayoría de estos autores, no obstante, y a fin de responder a algunas de las críticas clásicas, cabe puntualizar algunos términos. En primer lugar, la mayoría de ellos no mantienen un criterio tan rígido respecto a los estados de memoria experienciales. No es necesario que un sujeto recuerde todas sus experiencias

\footnotetext{
${ }^{5}$ En "Mr. Locke's Account of our Personal Identity". En Essays on the intellectual Powers of man, 1785.
} 
pasadas para poder mantener su identidad sincrónica, basta con que aquellos estados estén relacionados de la forma adecuada con los recuerdos actuales del sujeto. Además, los estados de memoria no son el único indicador de la identidad de un sujeto, el resto de estados mentales son también tenidos en cuenta. Por lo tanto, un sujeto P es idéntico a un sujeto p1 si sus estados mentales están relacionados de la forma adecuada. Además, la cuestión de la permanencia o la identidad diacrónica, que convertía en un verdadero problema la objeción de Reid, es atenuada con un criterio de continuidad psicológica. Los estados mentales de un sujeto no han de ser idénticos respecto a los estados mentales de un sujeto en el pasado, basta con que sean continuos con ellos. Es decir, si los estados mentales de un sujeto P son de alguna manera continuos con algunos de los estados mentales de un sujeto P1 en un tiempo anterior, entonces $\mathrm{P}$ y $\mathrm{P} 1$ son el mismo sujeto.

La mayoría de autores que defienden un criterio psicológico de determinación de la identidad personal concuerdan en la necesidad de rebajar las condiciones que Locke impone. No es necesario que un sujeto recuerde todas las experiencias que recordaba en un tiempo anterior, basta con que los estados mentales de un sujeto $\mathrm{P}$ en un tiempo t esté relacionados de la forma adecuada con los estados mentales de un sujeto en un tiempo anterior y que exista continuidad entre ellos para aceptar que son el mismo sujeto.

Shoemaker, por ejemplo, siguiendo a Grice $^{6}$, defiende una perspectiva psicologista pero que vincula la relación y continuidad de los estados mentales a relaciones de dependencia causal, haciendo de esta manera compatible la doctrina neolockeana de la identidad con un funcionalismo reduccionista como explicación general de la mente. Estas estrategias consiguen, en mayor o menor medida, hacer frente al argumento de transitividad de Reid, pues si bien la continuidad es transitiva, la conectividad admite grados. P no necesita recordar las experiencias de $\mathrm{P} 1$ en el tiempo t1, basta con que un número suficiente de sus estados mentales estén causalmente conectados con los estados mentales de P1 y sean continuos con ellos.

\footnotetext{
${ }^{6}$ Grice adopta el concepto de Total Temporary States (TTS). Grice propone hablar de conjuntos de experiencias simultáneas causalmente relacionadas (TTS) en lugar de personas. Dicho tratamiento le permite, entre otras cosas, salvar el argumento de transitividad de Reid.
} 
El otro argumento mencionado contra las tesis lockeanas es el argumento de circularidad del obispo Butler. Para hacer frente a este argumento Shoemaker (si bien es Parfit quien más claramente expone esta idea en referencia directa a la crítica de Butler) prefiere hablar de q recuerdos. Estos q recuerdos son recuerdos de experiencias en general que no necesariamente han de proceder de experiencias del sujeto que las recuerda. Pueden ser, por ejemplo, recuerdos implantados o copiados en su cerebro. En palabras de Parfit:

Consider Jane and Paul. Jane has agreed to have created in her brain copies of some of Paul's memory-traces. After she recovers consciousness in the post surgery room, she has a new set of vivid apparent memories. She seems to remember walking on the marble paving of a square, hearing the flapping of a flying pigeons and the cries of gulls, and seeing light sparking on green water. One apparent memory is very clear. She seems to remember looking across the water to an island, where a White Palladian church stood out brightly against a dark thundercloud (....) Since she knows that she has received copies of some of Paul's memory-traces, she could justifiably assume that she may be quasi-remembering some of Paul experiences in Venice (Parfit, 1984: 106).

Según este texto, Jane puede tener recuerdos de las experiencias de Paul "desde dentro", incluidos sus aspectos fenomenológicos. Lo único que Jane necesita saber para extraer información es en qué circunstancias tuvo lugar la experiencia de Paul. Si es cuasi imposible recordar experiencias de otros, la identidad no contaría como relevante en relación al contenido del recuerdo, pudiendo tratar los recuerdos normales meramente como una parte de total de recuerdos posibles, concretamente aquellos, más usuales, en los que el sujeto de experiencia coincide con quien recuerda.

\section{§4. Algunas cuestiones nuevas. La división De Cerebros}

Otro de los problemas teóricos que se presentan al criterio psicológico de identidad personal es la denominada línea de bifurcación o de división de 
cerebros $^{7}$. Bernard Williams (1973) es quien inicia esta línea. Nos sugiere pensar en el hipotético caso de Charles. Un hombre normal que un buen día se despierta con un agudo caso de amnesia. No recuerda nada de su vida anterior ni reconoce a nadie relacionado con ella. Su carácter y aparente personalidad también han cambiado completamente haciéndolo prácticamente irreconocible como Charles. Sin embargo, sus recuerdos y notas de carácter sorprendentemente coinciden con los de Guy Fawkes, el histórico personaje que intentó volar la cámara británica. No se trata simplemente de un trastorno de Charles, no es que se crea Guy Fawkes, es que realmente tiene sus recuerdos. Tiene información de detalles que solo podría tener si hubiera sido él, y una perspectiva de primera persona, esto es, no recuerda tal o cual acción sino a él mismo realizándola.

Según el criterio psicológico tendríamos que admitir que, de alguna forma, Charles ya no está y que la persona que ahora habita su estructura física es Guy Fawkes, pues es con quien existe continuidad psicológica. Sus estados mentales están causalmente relacionados con los de Fawkes, etc. De por sí, el experimento mental ya es bastante sorprendente, pero no acaba ahí. Williams afirma que si dicha transformación es posible en Charles también puede ser posible en otro hombre, por simplificar cuestiones de similitud, etc., que Williams discute, pongamos que en su hermano Robert. En el siguiente paso del experimento mental ambos (Charles y Robert) se despiertan con los mismos recuerdos de Fawkes. El problema de la transitividad vuelve a aparecer, pues ambos "son" Guy Fawkes, pero es absurdo decir que son la misma persona pues implicaría que la misma persona ocuparía dos lugares al mismo tiempo, lo que es imposible.

Para Williams la identidad es una relación "one-one", y no una relación "one-multiple". Además, la identidad no es independiente del cuerpo. La postura de Williams es que al menos un criterio físico es condición necesaria de la identidad personal.

\footnotetext{
${ }^{7}$ Para una explicación más detallada de todas estas cuestiones respecto al criterio psicológico, así como de su influencia en Parfit, ver J. O. Benito Vicente (2003 y 2008). Exposiciones críticas parciales aparecen también en Parfit (1984), Noonan (1989), Wiggings (1992) y Perry (2002).
} 
Muchas fueron las reacciones a este trabajo de Williams, aunque aquí solo vamos a exponer el trabajo de Shoemaker Persons and their Pasts (1970). En este trabajo Shoemaker nos pide imaginar a Brown y a Robinson, dos sujetos de características parecidas envueltos en un curioso experimento. Basándose en la posibilidad de un intercambio de cerebros, Shoemaker nos pide que imaginemos un experimento donde una vez más la ciencia permitiera la posibilidad teórica de conservar e intercambiar cerebros de un cuerpo a otro. En dicho experimento el cerebro de Brown, con todos sus recuerdos y demás estados mentales, es trasplantado en el cuerpo de Robinson, creando a la hipotética persona "Brownson". Según el texto de Shoemaker, los estados mentales de Brownson estarían causalmente relacionados de la forma correcta con las experiencias y estados mentales de Brown, por lo que a la hora de preguntarnos, si bien caben tres posibilidades: $a$ ) Brownson es Brown en el cuerpo de Robinson. b) Brownson es Robinson con el cerebro de Brown y c) Brownson es una persona completamente distinta a ambos, la única respuesta coherente sería la primera. Brownson es Brown. El criterio psicológico está a salvo pues, si bien es cierto que el cerebro es un órgano físico, su papel es meramente de soporte. Es la continuidad de la cadena causal entre las experiencias y recuerdos de una persona en el pasado y los estados mentales del presente lo que permite identificar a la "misma persona".

Bernard Williams, en su artículo "The Self and the Future" (1973), presenta otro punto de vista sobre el intercambio de cerebros. En dicho artículo Williams considera contrastar dos experimentos hipotéticos. En el primero escogerá a dos personas (A y B) y les explicará que sus cerebros van a ser intercambiados. Posteriormente les dirá que sus cuerpos van a ser uno premiado con cien mil dólares y el otro cruelmente torturado. Tras ser preguntados sobre sus preferencias al respecto, A decide que sea el cuerpo de A el torturado, pues piensa que él ocupará el cuerpo de $\mathrm{B}$ y recibirá la recompensa económica. B, obviamente, decidirá lo contrario. Tras el experimento, el cirujano ha decidido torturar el cuerpo de A. La persona A (en el cuerpo de B) se sentirá satisfecha de su buena elección, pues se ha respetado su preferencia y no ha recibido daño. La persona $\mathrm{B}$ (en el cuerpo de $\mathrm{A}$ ) se sentirá desafortunada porque no se ha tomado en cuenta su elección y sufrirá una cruel tortura. Sin duda hasta aquí todo parece indicar la superioridad de un criterio psicológico de la identidad. 
Sin embargo, a continuación Williams nos pide un esfuerzo más de imaginación. Esta vez solo hay una persona prisionera del malvado neurocientífico que le informa de que va a ser cruelmente torturada. Evidentemente las expectativas no son nada buenas. El malvado científico, no obstante, le informa de que justo antes de la tortura le será inducido un estado de amnesia total. $\mathrm{Ni}$ uno solo de sus recuerdos actuales se mantendrán en el momento de ser torturado. ¿Debe esto aliviar en algo a nuestro desgraciado personaje? Es evidente que no. Por mucho que se rompa la continuidad psicológica nuestro protagonista no dudará que será su cuerpo, y por lo tanto él mismo, el que será sometido a tortura. No obstante, se pregunta Williams, ¿qué diferencia encontramos entre el primer y el segundo caso? Una diferencia clara es que en el primer caso hay dos personas involucradas en el experimento, mientras que en el segundo hay solo una. Sin embargo, parece poco intuitivo tomar este dato como relevante para determinar la identidad personal. Más bien parece que la forma de presentación del problema determina nuestra respuesta.

Aún es posible una vuelta de tuerca más. Al parecer se han dado casos reales de personas que han sobrevivido con tan solo medio cerebro manteniendo intactas todas las funciones mentales. David Wiggins (2003) nos sugiere pensar en un caso más de división de cerebros. Wiggins rescata la posibilidad de dividir el cerebro en sus dos hemisferios a través de una intervención quirúrgica seccionando el cuerpo calloso. Al parecer dicha intervención se ha realizado con éxito en el tratamiento de ciertos casos de epilepsia. Como resultado de dicha intervención, al parecer, se producen dos corrientes independientes de conciencia.

Se ha dado algún caso de una persona que ha logrado sobrevivir con tan solo medio cerebro dada la capacidad del mismo de reorganizarse y adoptar las funciones de la otra mitad. Imaginemos que es posible seccionar un cerebro en sus dos mitades y trasplantar cada mitad en un cuerpo distinto. De nuevo tenemos un problema de bifurcación complejo. Por una parte es difícil aceptar que los dos nuevos individuos son idénticos, pues ocupan un espacio distinto y tan pronto despierten desarrollarán corrientes independientes de conciencia. Sin embargo se produce aquí una situación paradójica respecto al sujeto original. Cuando solo sobrevive medio cerebro no parece haber dudas respecto a la supervivencia pues, dada la capacidad del cerebro a adoptar 
funciones de la parte extirpada, los estados del sujeto resultante serán continuos con aquellos del sujeto original. Además estarían conectados de la forma correcta a ellos, cumpliendo las exigencias del criterio psicológico. No obstante, si ambas mitades del cerebro sobreviven implantadas en cuerpos distintos la identidad original parece perderse al dividirse en dos cursos de conciencia diferentes.

Resulta del todo paradójico convertir un doble éxito en el fracaso de la supervivencia del sujeto original al tiempo que aceptar la supervivencia de una conciencia en dos ramificaciones presenta toda una marea de problemas teóricos que no vamos a tratar aquí ${ }^{8}$. En su lugar vamos a explicar el tratamiento original que Parfit hace de este y otros experimentos y sus conclusiones sobre estos.

\section{§5. Derek Parfit: Lo QUe importa, La disolución Del yo Y SUS IMPLICACIONES MORALES}

Pero ninguno de los tratamientos de la identidad personal desde un enfoque lockeano es tan sorprendente en sus conclusiones como el de Derek Parfit. En Reasons and Persons Parfit realiza toda una serie de críticas sobre lo que denomina "éticas del interés propio". Ello le llevará a plantear la cuestión de la identidad y de qué tipo de ética justifica. De nuevo, como en Locke, la cuestión aparece vinculada a una definición forense.

Parfit parte de un enfoque donde se enfrentan dos visiones de la identidad personal. La que podemos denominar "la visión simple" (the simple view), que identifica la identidad personal con un ego cartesiano. Una entidad sustancial separada y distinta de los estados físico y psicológico que sostiene y por ello denominada también "teoría del hecho suplementario". Y la que denomina "la visión compleja" (the complex view), según la cual la identidad personal es determinada por otros estados físicos o psicológicos. Para Parfit, la visión simple supone una unidad de la conciencia en sentido fuerte, pero no

\footnotetext{
${ }^{8}$ Existe una interesante discusión sobre la conformación de un sujeto en partes temporales. Para más información ver Noonan (1989) y Perry (2002).
} 
sucede así necesariamente en la visión compleja. Esta conclusión tendrá implicaciones en el ámbito de la ética, lo que reforzará una determinada manera de entender la obligación moral, a saber, la propia del utilitarismo.

Parfit piensa que la mera continuidad en un cuerpo no es condición necesaria ni suficiente para garantizar la identidad. Su análisis de los ejemplos anteriormente expuestos y otros que son analizados en Reasons and Persons así parece establecerlo. Nuestro autor se sitúa en una posición que él mismo denomina "Reduccionismo" y adopta, dentro de este, un enfoque psicológico. Dos aspectos son centrales en la obra de Parfit; lo que llama conectividad psicológica y la continuidad entre los distintos estados mentales. Dos estados mentales están conectados cuando existe entre el primero en un tiempo y el segundo en un tiempo posterior algún tipo de relación causal del tipo adecuado. Si hay muchas conexiones de ese tipo, los estados están fuertemente conectados. Si existen, además, cadenas superpuestas de conectividad fuerte existe lo que llamamos continuidad. A dicha relación, establecida a través de la conectividad y la continuidad, Parfit la llamará la Relación R.

Una teoría de la identidad personal debe dar una respuesta adecuada a la cuestión que se planteaba en los ejemplos anteriores de división de cerebros en términos de "lo que importa para la supervivencia". En vista a proporcionar una respuesta, Parfit diseña su famoso experimento mental del teletransporte. Imaginemos que disponemos de la tecnología que lo hace posible y que hemos de viajar a Marte a través de este medio. Entramos en la cabina de teletransporte donde un potente escáner realiza un barrido de nuestra estructura atómica y la envía a Marte. Nuestro cuerpo es desintegrado al tiempo que una copia exacta de nosotros aparece en el planeta rojo. En tanto que los estados mentales de la copia son continuos con los nuestros, según el criterio psicológico tendríamos que aceptar la identidad de dicho individuo con nosotros.

Es evidente que dicha situación incomodará a muchos defensores del criterio físico para quienes, sin duda, el individuo de Marte es otro y el original no ha sobrevivido. Sin embargo, Parfit pone a prueba esta intuición modificando la primera versión del experimento. Esta vez nos pide imaginar que al viajar la máquina sufre una avería y funciona de forma inadecuada. Tras someternos al escáner nuestra copia aparece en Marte continua a nosotros. Sin embargo nuestro original no es destruido en la tierra. Estamos en uno de esos casos de 
bifurcación que como hemos visto más arriba tanto complican la cuestión. Más tarde, tras unas pruebas rutinarias, somos informados de que el fallo en la máquina no ha sido inocuo para nosotros. Al parecer su mal funcionamiento ha afectado de forma fatal nuestro corazón que fallará, irremediablemente, en unos días. Nuestra copia nos tranquiliza con la promesa de seguir con nuestra vida, cuidar de los nuestros, realizar nuestros proyectos, etc.

Según Parfit, aunque dicha situación no es equiparable a seguir con vida, tampoco podemos decir que es del todo equivalente a una muerte cierta, pues al menos "lo que importa" permanecerá. Dado que al existir bifurcación es evidente que el individuo de Marte no es idéntico a nosotros, la identidad no parece ser lo que importa. Lo que importa parece ser reducido a aquello que permanece: la Relación R. Como hemos visto, Williams asegura que la identidad es una relación one-one. Dado que lo que importa no es una relación de este tipo, lo que importa no es la identidad. Al parecer, según Parfit, este y otros ejemplos muestran que la pregunta acerca de la identidad es una pregunta que a veces carece de sentido.

En Later Selves and Moral Principles, Parfit ya insistía en esta idea de un sujeto no determinado comparando la identidad de una persona con la de una nación. La idea es que el concepto de nación, aunque trasciende el mero conjunto de personas que la componen y sus historias, sin embargo no supone nada suplementario a estas personas que la constituyen. Así, la pregunta de si Inglaterra es la misma nación en el siglo xi que en la actualidad, es una pregunta vacía de sentido.

Parfit establece así una concepción del individuo donde la conectividad admite grados. Desde lo que llama "complex view" no toda conectividad tiene la misma fuerza, igual que no todos los hechos históricos ni las personas que los llevan a cabo son igual de relevantes en la historia de una nación.

It Will seem like the truth that all the parts of a nation's history are as much parts of his history. Because this latter truth is superficial, we at times subdivide such a history into that of a series of successive nations, such as anglo saxon, Medieval or post imperial England. The connections between these, though similar in kind, differ in degree. If we take the complex view, we may also redescribe a person's life as the history of a series of succes- 
sive selves. And the connections between these we shall also claim to be similar in kind, different in degree (1973: 142).

Esta concepción del sujeto, cuya identidad no está determinada y que centra la atención de lo que importa no en un sujeto (hecho suplementario), sino en un conjunto de experiencias y otros estados $\mathrm{R}$ relacionados es mucho más débil que la concepción que conllevaría una teoría de la identidad personal desde lo que llama "the simple view", y esto tiene implicaciones a la hora de abordar la cuestión de la obligación moral. Puesto en cuestión el sujeto, solo algún tipo de consecuencialismo sobre el global de las experiencias tiene sentido para él.

Desde un punto de vista judicial, la "complex view" es fácilmente intuitiva. Es bien conocido que, desde la perspectiva lockeana, si alguien no recuerda haber cometido un crimen, entonces no es la misma persona que lo cometió y no puede ser condenada. Una consecuencia que a menudo, como recuerda Parfit, ha sido juzgada como "moralmente repugnante" (1973: 144). Sin embargo, el carácter parece algo más determinante que la mera memoria. Si las conexiones ente el autor del crimen y el actual individuo son lo suficientemente débiles, a medida que esa debilidad es mayor parece obvio que su responsabilidad va difuminándose. Si la conectividad es extremadamente débil o nula, el autor puede incluso ser inocente del crimen cometido. Cualquier tribunal atiende a atenuantes como "enajenación mental transitoria” etc. Conceptos como el de rehabilitación son también compatibles con esta concepción débil de la identidad.

Parfit confirma que el punto de vista al que llama "complex view" parece fortalecer una concepción utilitarista de la ética. Su contrapunto será la concepción de justicia distributiva, en especial desde la crítica al utilitarismo de Rawls.

Imaginemos una situación en la que un niño ha de soportar una fuerte carga que supondrá un importante beneficio en el futuro. Desde una perspectiva distributiva es importante de cara a la justicia que dicho beneficio recaiga sobre la misma persona que acepta la carga. Desde una perspectiva utilitarista lo importante es el beneficio mismo independientemente del para quién. Si aceptamos el punto de vista de Parfit, la conectividad débil entre los 
estados mentales del niño se hará más débil en el adolescente y casi nula en el individuo adulto. De esta forma, la perspectiva distributiva, que prima el "a quién" al "cuánto", perdería sentido o sería redundante. Este tipo de argumento es fácilmente trasladable a otras formas de entender la obligación moral. Aparentemente Parfit vincula prácticamente cualquier forma de moralidad, excepto el utilitarismo, al "simple view".

\section{§6. Dos modelos críticos con Parfit: HeRmenéutica E IDENTIDAD NARRATIVA Y AGENCIALIDAD}

Hemos visto como el debilitamiento de la idea de persona en Parfit conlleva determinadas consecuencias éticas. Unas consecuencias que conducen a un utilitarismo donde la intención moral no recae sobre las personas sino sobre un concepto concreto de beneficio y utilidad en general en un futuro. Como él mismo dice, no importa sobre "quién" sino "cuánto". Sin embargo, respecto a la identidad, no es fácil contentarse con ese planteamiento. Francamente, resulta llamativa la tranquilidad con la que Parfit asegura que, en su ejemplo del teletransporte, el hecho de que su doble le prometa seguir con su vida y sus proyectos le hace pensar que "su muerte" no será tan mala como "la muerte" pues lo que importa, esas cadenas superpuestas de conectividad fuerte, la relación $\mathrm{R}$, se mantendrá.

No deja de ser sorprendente para muchos críticos la rotunda afirmación de Parfit de que, en caso de sobrevivir un duplicado, la situación no puede equipararse del todo a la muerte. Claro que es igual que la muerte. Lo es porque lo que importa no es que mi conciencia encuentre continuidad o no, sino que esa continuidad sea "mi" continuidad". Da la impresión de que los argumentos de Parfit, aunque muy interesantes como crítica a ciertas concepciones del sujeto, así como una forma brillante de disolver ciertas intuiciones falsas acerca de la identidad personal, no acaban, sin embargo, de responder a la potencia intuitiva de que al final siempre buscamos a un sujeto de acción, a un sujeto

\footnotetext{
${ }^{9}$ Un argumento similar, centrado en la identidad personal y más extendido, podemos encontrarlo en J. O. Benito Vicente (2003).
} 
responsable de sus actos que dé cuenta de sus decisiones morales. El niño del principio de este artículo puede haber cambiado mucho cuando de adulto encuentra la carta. Desde luego la conectividad de sus estados mentales se habrá debilitado mucho en el trascurso de los años, pero fue él el que la escribió. El adulto que la encuentra se reconoce en ella, despierta determinados afectos y sensaciones que no se producirían de no ser él mismo quien la escribió.

Parfit piensa que todo lo que no sea "the complex view" acaba irremediablemente siendo cartesianismo. Tengo mis dudas de que la propia concepción de Parfit, como trataré de argumentar al final de este artículo, en tanto que heredera de Hume, no sea en última instancia una concepción cartesiana. De todas formas existen otros enfoques de la identidad personal y de sus implicaciones éticas que son críticas con Parfit y que están muy lejos de "the simple view". Vamos a esbozar brevemente dos de ellas y trataremos de sacar conclusiones.

\subsection{Hermenéutica e interpretación en el Sí Mismo como Otro}

En su obra Sí Mismo como Otro, Paul Ricoeur dedica su quinto estudio a la cuestión de la identidad narrativa (1996: 106 y ss.) y escoge como antagonista, precisamente, a Derek Parfit. En dicha obra Ricoeur realiza una distinción entre mismidad, que se opone a desemejanza, e ipseidad, que acude a la alteridad y se preocupa del cuidado de sí. No vamos a exponer aquí la compleja e interesante hermenéutica del sí que propone Ricoeur, pues eso excedería con creces lo propuesto en este trabajo, pero sus críticas a Parfit son, a mi entender, muy reveladoras y vale la pena tenerlas en cuenta.

En primer lugar Ricoeur se plantea la concepción que tiene Parfit de "reduccionismo". Ya en la lección anterior, dedicada al sujeto de acción y donde se confrontaba con Davidson, Ricoeur distinguía entre una ontología del estado y otra del acontecimiento. Mientras que el estado precisa de un sustrato que se encuentre en tal o cual estado, el acontecimiento no necesita más que un medio físico o psicológico en el que ocurrir. Para el filósofo francés, cuando Parfit acude al acontecimiento está ya planteando, desde el vocabulario que le es propio, el reduccionismo como tesis de referencia, delimitando así 
cualquier otra postura no reduccionista primero como parasitaria de esta, y en tanto que no reduccionista, como cartesianismo. En efecto, cualquier noción de persona no planteada en términos de ocurrencias físicas o mentales causalmente relacionadas entre sí y que tienen lugar en un cerebro supondrá un hecho suplementario a esta descripción de tercera persona. Hecho suplementario que Parfit encuentra, en su formulación más evidente, en la sustancia espiritual cartesiana.

Sin embargo, Ricoeur piensa que en realidad, cuando Parfit hace referencia a esta sustancia, está, al no tomar en cuenta la dualidad entre mismidad e ipseidad, renunciando precisamente a aquello que Ricoeur llama "calidad de mío", y que no aparece en una descripción dada en el vocabulario de la facticidad (1996: 130). Ahora bien, es mi relación con mi cuerpo, con mi existir en él, aquello que me hace tener mis experiencias como propias. El dualismo que plantea Parfit no es, pues, un dualismo entre sustancias, espiritual y física, sino un dualismo entre pertenencia mía y descripción impersonal. En última instancia, piensa Ricoeur, la confrontación es entre dos concepciones del cuerpo. El cuerpo como mío y el cuerpo como cualquier cuerpo. Es por ello, según Ricoeur, que la ontología del acontecimiento sitúa en el cerebro el discurso del cuerpo, pues de todos los órganos es el "menos vivido"10.

En cuanto a los estados psicológicos Ricoeur pone el acento en la recurrencia de Parfit a la memoria. Recordemos que, en vistas a sortear el argumento de circularidad de Butler, Parfit propone tratar los estados de memoria como un tipo especial de memoria donde es posible tener recuerdos implantados de experiencias de otros. Pero "¿puede lo propio ser un caso particular de lo impersonal?". Ricoeur piensa que necesariamente hay pérdida semántica cuando sustituimos "yo pienso" por "esto piensa" (o "el pensamiento está en curso"). Existe una diferencia cuando adscribimos un pensamiento a nosotros mismos o a otros que irremediablemente se pierde en una descripción impersonal. De hecho, aunque Ricoeur no lo apunte como tal, lo que permite

10 "Solo mediante el rodeo global por mi cuerpo, y que el cerebro está contenido en este cuerpo, puedo decir: mi cerebro. El carácter desconcertante de esta expresión se halla reforzado por el hecho de que el cerebro no entra en la categoría de los objetos percibidos a distancia del propio cuerpo. Su proximidad en mi cabeza le confiere el extraño carácter de interioridad no vivida", ibíd., p. 131. 
precisamente pensar la memoria es el tener acceso a experiencias de otros "como mías".

Otro punto interesante también en el Sí Mismo como Otro es la comparación que Ricoeur hace entre la ficción narrativa y la ciencia ficción de los ejemplos de la filosofía analítica en general, que antes hemos mencionado, y los de Parfit en particular. Estas ficciones, en especial la del teletransporte, son utilizadas por Parfit para argumentar que la identidad personal no es determinable. No siempre tiene sentido preguntar: “¿Voy a sobrevivir?”.

La identidad ha sido siempre un campo de pruebas para las aporías y paradojas. Desde cuestiones bien tempranas se ha puesto a prueba el recurso a la imaginación para crear situaciones de difícil determinación. El tipo de ficción al que recurre la filosofía analítica tiene ya su inicio en el propio Locke. Casi todas las ficciones filosóficas empleadas recientemente tienen su origen en alguno de los ejemplos que aparecen en el Essay. El propio Ricoeur acabará contraponiendo su propia ficción, en este caso literaria, a la ficción científica de los analíticos.

Una vez más, no obstante, Ricoeur señala que el necesario corolario a estos ejemplos aparece ya predeterminado en el tipo de elección de la ficción que el autor realiza. La mayoría de estas ficciones ocurren en el ámbito no de lo realizable, sino en el de lo lógicamente posible en el contexto de una tecnología futura. La posibilidad de la ciencia de manipular, seccionar y dividir el cerebro, órgano predilecto donde ubicar la mismidad por su anteriormente nombrada cualidad de "órgano no vivido", predibuja ya una conclusión. Para Ricoeur el problema de la ipseidad ha sido eliminado por principio. En efecto, en los casos límites mencionados más arriba, y desde los presupuestos imaginativos de la ficción científica, parece difícil sustraerse a la aporía que resulta a la hora de elegir entre (1996: 133):

- No existe nadie que sea el mismo que yo.

- Yo soy el mismo que uno de los dos individuos surgidos de la experimentación.

- Yo soy el mismo que los dos individuos. 
En este sentido la paradoja se revela como una paradoja de la mismidad. Una paradoja que surge de separar en la ficción lo que en la experiencia común es indisociable, a saber; aquello que es susceptible de una descripción impersonal entendido como una urdimbre causal de estados psicológicos y el propio sentimiento de pertenencia a alguien que se concibe a sí mismo como poseedor de esos estados.

Mientras tanto algo ocurre. Ricoeur nos recuerda que en el mismo ejemplo del teletransporte aparece como insuperable la cuestión de la temporalidad. Una temporalidad que no aparece en el ámbito de la descripción impersonal de las condiciones del propio viaje, pero que se revela como indispensable en el viajero. ¿Voy a sobrevivir? Es la pregunta fundamental que este se hace. En los términos de mismidad en que el experimento es diseñado esta pregunta queda sin respuesta, pero en el deslizamiento de la cuestión de las relaciones de los procesos de memoria a la cuestión de la supervivencia aparece una historicidad de un sujeto que se anticipa y se preocupa. El viajero duda, cree, teme y se pregunta si va a sobrevivir. Esta dimensión es muy difícil de atrapar en una descripción impersonal y, por mucho que la ficción científica trate de eludirla, esta reaparece en los términos mismos de un preguntarse cuya respuesta es, ya prefigurada, una aporía de la mismidad.

Esta misma dificultad aparece también en el tratamiento que hace Parfit de la superación de la aporía misma. La identidad aparece como no determinable, por lo tanto no es "lo que importa". Lo que importa es la relación R. Parfit parece plantear la cuestión como un lo que importa para qué, si bien Ricoeur cree que no puede sustraerse a lo que importa para quién.

La cuestión de la indecibilidad nos es inaceptable. Por eso rechazamos dentro de los planteamientos de Parfit, la identidad como aquello que importa, porque nos turba, nos molesta.

Es importante recordar aquí que el trasfondo de la cuestión es, en la filosofía de Parfit, una cuestión ética. El oponente de Parfit es el teórico del interés propio. Parfit trata de demostrar que el sujeto de dicho interés es un sujeto de una ontología débil e indeterminada que no ofrece, en realidad, diferencia alguna entre sus distintos yoes y entre los otros. La elección ética desde semejante concepción del sujeto no puede evitar ser vacía si tomamos 
como criterio el a quién, pues ese quién aparece más o menos difuminado en una serie de $\mathrm{R}$ relaciones en las que este está ausente o es meramente redundante. Es por ello que el trabajo de Parfit respecto al yo parece reforzar al utilitarismo como el único planteamiento ético racional al preocuparse por la cantidad de beneficio sin preguntarse por el a quién va dirigido ese beneficio. La diferencia entre que ese beneficio, fruto de cargas presentes, vaya a un yo futuro débilmente $\mathrm{R}$ conectado conmigo o a otro es meramente una distinción lingüística sin interés ético alguno.

Sin embargo, ¿a qué yo se está refiriendo Parfit? Como hemos visto, tanto las definiciones como los argumentos y ficciones encaminadas a demostrar la indeterminación del yo, y por tanto su no pertinencia, se centran en lo que Ricoeur denomina mismidad. Si soy yo ese yo es una pregunta que puede no tener siempre una respuesta. Sin embargo, cuando habla de elección ética, cuando nos planteamos la cuestión de lo que importa, lo que nos planteamos es la cuestión de la ipseidad indirectamente eludida por el planteamiento reduccionista desde su inicio, y que se revela en el análisis de Ricoeur como insalvable. "Lo que importa" es una expresión incompleta, pues ¿a quién le importa? De lo que se trata es de la resistencia del quién en todos los análisis del qué (Ricoeur, 1996: 136). Explicado muy brevemente, en efecto, no me importa ahora qué estructura neuronal sosteniendo qué entramado psicológico tenga en el futuro, pues puede estar muy débilmente conectada con dicho entramado la persona que soy ahora, es cierto, pero sí me importa que el beneficio de mis cargas presentes sea para "mí'.

Como he mencionado anteriormente, no me interesa en este trabajo profundizar en la hermenéutica del sí de Ricoeur, pero resulta muy revelador cómo su distinción entre mismidad e ipseidad pone al descubierto carencias importantes en la tesis de Parfit. Estas carencias no dejan de tener también implicaciones éticas que han sido fruto de la reflexión de otros autores. Vamos a continuación a tratar de exponer el trabajo de una de las filósofas que plantea la cuestión del sujeto y la ética desde una perspectiva muy diferente a la de Parfit. Aunque el planteamiento en sí mismo es muy diferente también al de Ricoeur, las debilidades a las que alude en el planteamiento analítico son las mismas o muy similares a las reveladas hasta ahora por el filósofo francés. 


\subsection{Agencialidad y sujeto práctico en Christine Korsgaard}

En Personal Identity and the Unity of Agency Christine Korsgaard propone un planteamiento kantiano del sujeto y la ética sobre la base de la crítica al planteamiento de Parfit. Según Korsgaard (1989), las implicaciones del sujeto en ética no son epistemológicas sino prácticas. El utilitarismo, tal como defiende Parfit, no establece una relación especial de mi yo con el yo del futuro, lo que permite un tratamiento impersonal de la ética en términos de mayor beneficio posible. Sin embargo, cuestiones como el compromiso moral con la palabra dada o la responsabilidad para conmigo mismo en el futuro descansan en una relación lo suficientemente débil para, incluso teniéndola en cuenta, no ser determinante en mis elecciones.

El planteamiento de Parfit es muy similar al de Hume. Si partimos de una perspectiva epistemológica todo lo que puedo llegar a averiguar de mí mismo son un conjunto de experiencias y disposiciones que me inclinan a actuar de una u otra manera. Un conglomerado de experiencias, como dice Hume. Korsgaard recuerda la distinción kantiana entre pasividad y actividad del sujeto racional. Desde la perspectiva de Parfit, los aspectos activos, las decisiones que tomamos, etc. son reducibles a meras experiencias, a cosas que nos pasan, a disposiciones que pueden ser traducidas y expresadas también en un lenguaje impersonal. Su análisis de la promesa dada, tanto en Later Selves como en Reasons and Persons así lo ponen de manifiesto.

Korsgaard, no obstante, cree que Parfit no toma en consideración el hecho mismo de la decisión. En este sentido se pregunta qué ocurre cuando existen varios motivos para actuar de una u otra forma que entran en conflicto. Parfit toma como ejemplo en el tratamiento de esta cuestión la división de un cerebro en dos cursos paralelos de conciencia que mencionábamos arriba. Si la información de ambos cursos de conciencia es contradictoria no tendremos una decisión, sino dos. Ambos hemisferios cerebrales darán una orden contradictora respecto al otro, mostrándose así ineficaces. Para Korsgaard, sin embargo, es necesario en la acción tomar una decisión, somos un sujeto porque tomamos decisiones sobre cómo actuar, y solo tenemos un cuerpo con el que actuar. 
Parfit, en el ejemplo mencionado, argumentaría que, en caso de conflicto, existirán razones que decidan qué parte del conflicto es más relevante, cuál aportará mayor beneficio en el futuro, y esta se impondrá. Sin embargo, resulta poco intuitivo pensar que todo lo que hacemos es esperar que se resuelva una situación de conflicto y observar cómo actuamos. Parece más bien que hacemos algo, decidimos incluso por encima de la conveniencia o no de los motivos y lo hacemos "nosotros". De esta manera, la unidad y determinación de un sujeto puede no ser necesaria como cuestión metafísica, pero se adivina fundamental en la cuestión práctica. Hay un sujeto que elige tomar esta o aquella decisión y que, al tomarla libremente, es responsable de ella. Un planteamiento que bien podríamos encontrar en el Kant más práctico.

Como explicamos con anterioridad, Parfit compara la unidad de una persona con la unidad de una nación. No hay un hecho suplementario a una nación más que sus habitantes. Sin embargo, las relaciones entre estos habitantes no son más determinantes en la identidad de la nación que los estados de experiencia lo son de la identidad de una persona. Las relaciones son débiles, y no siempre tiene sentido la pregunta por la identidad. Korsgaard, por contra, piensa que desde el supuesto práctico la comparación con una nación es insuficiente e incluso caería en la falacia naturalista, pues trataría de explicar lo que debería ser desde lo que es. La comparación justa en este sentido no sería con una nación, sino con un estado. Un estado es mucho más que un conjunto de ciudadanos, pues precisa para serlo de aquello que precisamente los convierte en ciudadanos. Un estado es también lo que Korsgaard llama "una forma", una serie de requisitos formales que unifica y trasciende al mero conjunto, le da sentido y unidad y justifica su identidad a lo largo de la historia (Korsgaard, 1989: 112).

De la misma manera, la unidad de la conciencia necesaria que no encontramos en un enfoque epistemológico aparece exigida en la necesaria propositividad de un sujeto agente que debe actuar de acuerdo con unos criterios formales que den sentido moral a la elección, un sentido moral acorde con la exigencia de responsabilidad que pedimos al sujeto.

A menudo las acciones no son elecciones puntuales de las que cabe calcular su beneficio concreto en términos de ganancia o pérdida, sino que forman parte de un fin. Realizamos planes, establecemos metas, nos ocupamos 
de nuestro futuro, elegimos una carrera profesional, un modelo de forma de vivir, una estructura de vida a la que no es indiferente qué valores morales elegimos. Toda esa estructura requiere de un sujeto que unifique las diferentes acciones y decisiones en un marco formal que nos requiere como sujetos de propósitos.

La apuesta de Parfit es creer que un número indeterminado de agentes ocupará mi cuerpo a lo largo de mi vida y que estos llevan mis asuntos de igual manera que un grupo de trabajo puede funcionar como una unidad propositiva. Pero ¿agota ese análisis la necesidad formal de un sujeto? Podemos imaginar un territorio habitado por un grupo de personas que se coordinan para realizar ciertos trabajos que faciliten su vida en ese territorio. ¿Hace de ellos un Estado? La propositividad propia de un sujeto agente requiere, como argumenta Korsgaard, de una unidad que trasciende el conjunto de estados psicológicos de un sujeto y que lo dota de motivos e historicidad. En su trabajo Korsgaard trata de defender el concepto de justicia distributiva de Rawls. No es mi interés en este trabajo defender un modelo ético político u otro, sino mostrar que la apuesta utilitarista de Parfit deja muchas ventanas abiertas por las que se cuelan cuestiones de diversa índole moral.

\section{§7. CONCLUSIÓN}

Los planteamientos de Ricoeur y de Korsgaard son muy diferentes. La simplicidad corporal de la ipseidad choca con la complejidad del sujeto ético formal de Korsgaard, pues sus intereses finales están muy lejos. Pero ambos coinciden en su crítica fundamental a Parfit. Una concepción puramente descriptiva en términos de tercera persona de las experiencias del sujeto no recoge aspectos fundamentales del mismo para una adecuada comprensión del hecho moral.

Por recordar a Kant, Parfit parte del escepticismo que supone la negación de toda unidad más allá de lo susceptible a una descripción analítica e impersonal de la experiencia. Sin embargo, aunque su apelación a lo "impersonal" viene ya exigida en el planteamiento mismo, ¿es posible una descripción semejante de sí mismo que abarque el ámbito de lo práctico? Problemas moder- 
nos que vuelven a estrellarse con aporías modernas. Acabamos de explicar el planteamiento kantiano de Korsgaard, y un poco más arriba hemos explorado el enfoque hermenéutico de Ricoeur.

Ninguna interpretación de estos dos planteamientos se adecua a lo que Parfit llama "the simple view" o a teoría del hecho suplementario alguna. Como bien señala Ricoeur, las teorías analíticas del sujeto, y Parfit en particular, parten de una concepción ontológica de la facticidad que en la actualidad está siendo cuestionada desde diversos frentes ${ }^{11}$. El debilitamiento del sujeto en Parfit parte de una concepción del mismo que ha de construirse en el lenguaje propio de un reduccionismo que, lejos de superar la dualidad cartesiana, trata de explicar la conciencia desde una concepción desubicada de la facticidad como superadora de lo mental, lo que no ayuda a la ética.

Tratar de explicar una ética sin el otro, sin referencia alguna al ámbito social y de relación intersubjetiva en el que adquiere significación el hecho moral mismo deja efectivamente indeterminado y vacío al sujeto. Esta indeterminación no es nueva. Ya aparece en el propio Descartes que, si bien es el paradigma del dualismo, asume y da cuenta de la dificultad de los problemas que este plantea. Parfit analiza de forma brillante las consecuencias que conlleva el reduccionismo, un sujeto humeano, un conjunto de experiencias $\mathrm{R}$ relacionadas que parecen no justificar más ética que la utilitarista cuyos receptores no son sujetos concretos sino el conjunto global de experiencias sobre las que recaen las consecuencias de la acción.

Sin embargo, cabe preguntarse si realmente nuestra concepción de la ética, nuestras intuiciones, acciones y valores caben en una explicación como esa o si por el contrario, la cuestión que Ricoeur llama ipseidad vuelve a aparecer una y otra vez exigiendo a los hechos su preocupación acerca de lo que verdaderamente importa. Al final, tanto mi preocupación moral como mía, como la responsabilidad hacia el otro, aparecen ineludiblemente como previas a cualquier interpretación descriptiva del hecho moral, mas nunca lo hacen en el propio contenido.

\footnotetext{
${ }^{11}$ Podemos recordar también planteamientos narrativos como el de MacIntyre y las llamadas éticas de la virtud, o comunitarismos como el de Taylor y otros. Ninguno de estos planteamientos respondería a una concepción del sujeto desde lo que Parfit denomina "the simple view". Simplemente parten de una concepción distinta a la propia de la facticidad.
} 
Desde mi punto de vista, Parfit no puede superar la necesidad de apelación a la unidad de la conciencia por la misma razón por la que los empiristas modernos no pudieron. La resistencia a la reducción de un "yo" que acompaña todas mis experiencias, pero que necesariamente no aparece en el contenido de las mismas. La naturaleza misma de ese yo es en sí problemática, desde luego. Muchos enfoques a lo largo de la historia más o menos reciente de la filosofía así lo atestiguan, pero está ahí, y no puede sin más pasarse por alto. Por otra parte, la necesaria vinculación de la concepción del sujeto con la ética puede formar parte de una concepción de la moral que tanto Locke como Parfit parecen tratar de rodear sin éxito.

\section{REFERENCIAS BIBLIOGRÁFICAS}

Benito Vicente, J. O. (2003). El problema de la identidad personal en la filosofía analítica. Daimon (28), 67-83.

Benito Vicente, J. O. (2008). Racionalidad e Identidad: El problema de la identidad personal en Derek Parfit. Tesis Doctoral. Valencia: Universitat de Valencia.

Grice, H. P. (1941). Personal Identity. Mind (200), 330-350.

Hume, D. (1988). Tratado de la Naturaleza Humana. Madrid: Tecnos.

Korsgaard, C. (1989). Personal Identity and the Unity of Agency: A Kantian Response to Parfit. Philosophy and Public Affairs, 18(2), 101-132.

Locke, J. (1961). An Essay Concerning Human Understanding. London: Ed. J. Yolton. Versión Española (2005). Ensayo sobre el Entendimiento Humano. México D. F.: Fondo de Cultura Económica.

Noonan, H. (1989). Personal Identity. London: Routledge.

Parfit, D. (1971). Personal Identity. The Philosophical Review, 80(1), 3-27.

Parfit. D. (1973). Later Selves and Moral Principles. En A. Montefiori (ed.), Philosophy and personal relations. London: Routledge and Kegan Paul.

Parfit, D. (1984). Reasons and Persons. Oxford: Clarendon Press. Versión española (2004). Razones y Personas. Madrid: A. Machado Libros. 
Perry J. (2002). Identity, Personal Identity and the Self. Hackett Publishing Company.

Ricoeur, P. (1996). Sí Mismo como Otro. Madrid: Siglo XXI Editores.

Shoemaker, S (1963). Self Knowledge and Self Identity. Ithaca, Cornell University Press.

Shoemaker, S. (1970). Persons and Their Pasts. American Philosophical Quarterly, 7(4), 269-285.

Shoemaker, S. (1996). The first Person Perspective, and other Essays. Cambridge University Press.

Taylor, Ch. (1996). Las Fuentes del Yo. Barcelona: Ediciones Paidós Ibérica.

Wiggins, D. (1980). Sameness and Substance. Cambridge, Massachusetts: Harvard University Press.

Wiggins, D. (1992). Remembering Direcly. En Hopkins, Savile (eds.), Pychoanalysis, Mind and Art. Oxford: Blackwell.

Wiggins, D. (2003). Sameness and Substance Renewed. Cambridge University Press.

Williams, B. A. O. (1970). The Self and the Future. The Philosophical Review, 79(2), 161-180.

Williams, B. A. O. (1973). Problems of the Self. Cambridge: Cambridge University Press. 